\title{
Um estudo sobre o perfil das equipes de desenvolvimento de softwares educacionais
}

\author{
Leandro Veloso Rodrigues, Sérgio A. Andrade de Freitas, Fabiana Freitas Mendes \\ Faculdade UnB Gama (FGA) - Universidade de Brasília (UnB) \\ Área Especial de Indústria Projeção A, Setor Leste - Gama \\ 72.444-240 - Brasília - DF - Brazil \\ leandrovelosorodrigues@gmail.com, \{SergioFreitas,FabianaMendes\}@unb.br
}

\begin{abstract}
Non-technical competencies associate knowledge, attitudes and skills in distinct areas such as social, personal and cognitive. These skills are important for the success of any software project. Besides, regarding the development of educational software they are even more fundamental. Such competencies have not been clearly observed in Computer and Engineering graduates, which are primary source of software development professionals. In this sense, this paper explores whether the Software Engineering graduates, who engaged in the Ciencia sem Fronteiras exchange program, are developing such skills in other conditions.
\end{abstract}

Resumo. Competências não técnicas relacionam conhecimentos, atitudes e habilidades associadas às diversas áreas: social, pessoal e cognitiva. Essas competências são importantes para o sucesso de qualquer projeto de software. Entretanto no desenvolvimento de softwares educacionais elas são fundamentais. Tais características não têm sido claramente observadas nos egressos dos cursos da área de Computação e da Engenharia, fontes primárias de profissionais para desenvolvimento de software. Neste sentido, este trabalho explora se os egressos de um curso de Engenharia de Software participantes do programa Ciência sem Fronteiras estão desenvolvendo tais competências em outras condições.

\section{Introdução}

Competências não técnicas relacionam conhecimentos, atitudes e habilidades associadas às diversas áreas: social, pessoal e cognitiva. Essas competências são igualmente importantes para o sucesso de qualquer projeto de software quanto às competências. Estas características, muito das vezes interpessoais, são cada vez mais necessárias nas equipes técnicas de desenvolvimento de softwares educacionais (Neto, Reinehr, \& Malucelli, 2015).

Tipicamente, as equipes de desenvolvimento de software têm sua formação realizada em cursos de Computação ou Engenharia, onde os estudantes são capacitados na solução técnica de problemas. Não havendo a preocupação, na maioria das vezes, com outros aspectos não técnicos da formação do profissional. Estas competências apesar de estarem preconizadas nas Diretrizes Curriculares Nacionais (DCNs) para os cursos de Engenharia (Ministério da Educação, 2002) e para os cursos de Computação (Ministério da Educação, 2012), não são claramente observadas nos egressos destes cursos. Uma exceção são os cursos de Licenciatura em Informática cujo cerne da 
V Congresso Brasileiro de Informática na Educação (CBIE 2016)

Anais do XXVII Simpósio Brasileiro de Informática na Educação (SBIE 2016)

formação está na área de Educação. As razões são diversas e fogem ao escopo deste artigo.

Posto este cenário, a questão aqui tratada é investigar se existem outras formas de capacitação destes estudantes nos aspectos não técnicos necessários no desenvolvimento de softwares educacionais: sensibilidade cultural, sensibilidade social, comunicação oral e escrita, habilidade de trabalhar em equipe entre outros. De forma mais especifica, investigar se esta formação (Santos, Magalhães, Correia-Neto, Souza, \& Vilar, 2014) pode ser obtida por estudantes de um curso de Engenharia de Software quando do seu estágio em outros países através do Programa Ciências sem Fronteiras $(\mathrm{CsF})$.

A execução desta pesquisa foi realizada em três fases: primeiramente, foi feito um trabalho de revisão bibliográfica sobre a formação em Engenharia de Software, com o levantamento dos aspectos não técnicos preconizados na literatura. Numa segunda fase, seguindo o modelo de Pasquali (Pasquali, 2011), os aspectos foram transformados em indicadores e validados junto a um grupo de juízes (professores do curso). Os indicadores mais frequentes foram transformados em um questionário e aplicados a um grupo 48 estudantes do curso que participaram do CsF. Finalmente, na terceira fase foi feita a análise dos dados.

Esta pesquisa exploratória está assim estruturada: na seção 2 são apresentados os trabalhos correlatos, na seção 3 é feita uma revisão da literatura que embasa os questionários aplicados, na seção 4 é apresentado o levantamento e validação dos itens do questionário, na seção 5 é feita a análise dos resultados e, por fim, na seção 6 são apresentadas algumas conclusões e trabalhos futuros.

\section{Trabalhos correlatos}

Artigos e algumas dissertações que descrevem a experiência e efetividade do programa CsF foram publicados nos últimos anos. Todos estes estudos são relacionados a análises sob uma perspectiva de política pública ou ainda como estudo de caso de Instituições de Ensino Superior ou cursos de graduação (Barreto, Silva, Bezerra, \& Jesus, 2013). Não existe, no entanto, publicações que propõem a perspectiva neste estudo realizada. A Engenharia de Software com um olhar não técnico é o diferencial com relação aos trabalhos já realizados.

Para o desenvolvimento deste trabalho foi necessário a definição de uma lista com aspectos não técnicos que são fundamentais para um Engenheiro de Software. Esta lista não existe diretamente nos modelos e guias de Engenharia de Software (SWECOM, 2015; SWEBOK, 2014; Ministério da Educação, 2012; IEEE Computer Society and ACM, 2004). Dois trabalhos sobre este tema foram encontrados, ambas são publicações desenvolvidas em um contexto externo, fora do Brasil, assim, as competências não técnicas que são apresentadas pelos autores possivelmente não representam em sua totalidade o perfil dos engenheiros de software brasileiros (Lacher, et al., 2015; Rivera-Ibarra, Rodríguez-Jacobo, \& Serrano-Vargas, 2010).

Uma das publicações apresenta uma lista de competências, comportamentos e atitudes que foi extraída e validada com rigor através de uma revisão sistemática (Lacher, et al., 2015). A outra, por sua vez, é um framework obtido por meio de estudos empíricos realizados pelos autores em um período de sete anos. O foco da pesquisa era levantar as competências necessárias e relacionadas ao processo de desenvolvimento de 
V Congresso Brasileiro de Informática na Educação (CBIE 2016)

Anais do XXVII Simpósio Brasileiro de Informática na Educação (SBIE 2016)

software. A revisão bibliográfica foi utilizada como suporte teórico para as observações realizadas, porém nenhuma técnica de revisão em específico é mencionada no documento (Rivera-Ibarra, Rodríguez-Jacobo, \& Serrano-Vargas, 2010).

O trabalho de Lacher, et al. (Lacher, et al., 2015) foi utilizado como uma das fontes principais dos itens a serem validados neste estudo por descrever uma melhor estruturação e execução. Sua contribuição está descrita na Seção 4.

\section{Revisão de Literatura}

Esta seção apresenta uma visão geral sobre a teoria e conceitos que envolvem os tópicos tratados neste artigo.

\subsection{Ciências sem Fronteiras}

Instituído em dezembro de 2011 (Governo Brasil, 2011), o programa Ciências sem Fronteiras foi decretado como parte de um programa educacional do Governo Federal com iniciativa conjunta do Ministério da Educação e dos Ministérios da Ciência, Tecnologia e Inovação. Cada ministério possui uma instituição de fomento: o CNPq e a CAPES. Através de esforços destas duas instituições e das secretarias de ensino superior e de ensino tecnológico do ministério da educação, foi possível idealizar e concretizar o programa CsF.

O objetivo geral do programa é prover condições de uma formação dos estudantes brasileiros em um nível de elevada capacitação através do estudo em instituições internacionais. Complementar a este macro objetivo, no decreto oficial que institui o programa, estão listados nove objetivos específicos. A maioria com foco na ampliação da qualidade técnica e acadêmica brasileira, através da internacionalização do conhecimento. Assim, o governo almeja através deste programa e outras políticas públicas, um grande impacto que irrigue todo o sistema acadêmico nacional.

A estrutura do programa previa inicialmente a concessão de até 101 mil bolsas distribuídas em editais publicados entre 2011 e 2014. Foram concedidas 92.880 bolsas nas seguintes modalidades: Graduação Sanduíche, Doutorado Sanduíche, Doutorado no Exterior, Atração de Jovens Talentos, Pós-Doutorado no Exterior, Pesquisador Visitante Especial e Mestrado no Exterior. Essas sete modalidades de bolsas foram disponibilizadas para que os objetivos do programa fossem alcançados permeando desde a graduação até a pós-graduação e programas de extensão (Governo Brasil, 2014).

\subsection{Modelos e Guias da Engenharia de Software}

Apesar da engenharia de software, como termo e conceito, datar de 1968 (Wirth, 2008), a definição, estudo e produção de guias que propusessem uma grade curricular e um mapeamento das habilidades e competências desta área, foram tomados como objeto de estudo apenas nos últimos 15 anos. A estrutura dos cursos de graduação em ciências da computação passou a ser objeto de melhorias e estudos sobre a verdadeira contribuição profissional que a grade curricular padrão do curso proporcionava. No final da década de 90, empregadores apontavam que os egressos deste curso possuíam experiência insuficiente na participação e envolvimento nos projetos em equipe. Produzir de forma eficiente e entender os negócios e processos organizacionais, no âmbito de desenvolvimento, também estava aquém do esperado. Esta falta de conhecimento sobre 
V Congresso Brasileiro de Informática na Educação (CBIE 2016)

Anais do XXVII Simpósio Brasileiro de Informática na Educação (SBIE 2016)

equipe, metodologia e processos de software foi evidenciada com a alta na demanda de engenheiros de software.

A Engenharia de Software passou a ser moldada como uma área e curso de graduação. Muitos esforços foram e são desprendidos na criação de documentos e guias que permitam melhor defini-la. Diversas contribuições desenvolvidas pela IEEE-CS em relação ao amadurecimento da Engenharia de Software em termos curriculares, de conhecimento e também de competências foram publicadas nos últimos anos (IEEE Computer Society and ACM, 2004; SWEBOK, 2014; SWECOM, 2015).

\subsection{SE2004 e SWEBOK}

Em 2004 foi disponibilizado, pela IEEE-CS (IEEE Computer Society and ACM, 2004) em conjunto com a ACM, um volume relacionado à Engenharia de Software como parte de um projeto de orientações para currículos de graduação chamados SE2004. As orientações foram construídas em busca de um currículo que possuísse o balanceamento ideal das disciplinas de computação e engenharia.

O projeto é dividido em dez áreas de conhecimentos que são detalhadas em 41 unidades curriculares. Um breve corpo de conhecimento também está presente no guia. As informações deste corpo de conhecimento são melhores detalhadas no SWEBOK (SWEBOK, 2014). O SWEBOK é um documento estruturado em quinze áreas de conhecimento. Em cada capítulo os autores introduzem o conteúdo em tópicos e subtópicos mapeando-os em uma matriz que apresenta o referencial bibliográfico.

\subsection{SWECOM}

O SWECOM (SWECOM, 2015) é um modelo direcionado a uma variedade de propósitos. Ele promove um conteúdo sólido que apresenta as competências de um engenheiro de software separadas por áreas de habilidade e atividades, classificadas de acordo com o nível técnico de competência do profissional. Entre os modelos e guias selecionados para estudo neste trabalho, o SWECOM é o de mais recente disponibilização. O profissional que exerce a função de engenheiro de software deve possuir como resultado de um adequado currículo e de um corpo de conhecimentos adquirido pela sua vida educacional, um conjunto mínimo de competências técnicas e comportamentais. O SWECOM almeja prover um modelo contendo todas as competências esperadas para este profissional.

Todos os documentos acima descritos possuem como foco primário as competências técnicas da Engenharia de Software. Entretanto, uma breve menção as habilidades cognitivas, atributos comportamentais e profissionais pode ser extraída dos documentos.

\section{Levantamento dos indicadores}

Para a determinação de quais indicadores (competências) são esperados destes profissionais, uma adaptação de revisão sistemática foi realizada. A fonte de pesquisa, os critérios de seleção e de avaliação dos itens seguiram um conjunto de critérios préestabelecidos a fim de eliminar as possibilidades de viés e trazer resultados melhor definidos e relevantes ao estudo (Wohlin, et al., 2012). 
V Congresso Brasileiro de Informática na Educação (CBIE 2016)

Anais do XXVII Simpósio Brasileiro de Informática na Educação (SBIE 2016)

Apesar de todos os benefícios e vantagens associados à utilização de uma revisão sistemática, a natureza e foco do trabalho não são totalmente justificadores para a sua plena execução, uma vez que sua rigorosa especificação vai além da intenção deste trabalho. Assim, apenas as instruções e o modelo do protocolo foram adaptados e utilizados com o intuito de realizar de forma flexível e verificável uma revisão bibliográfica. Este método foi utilizado para a extração de informações das fontes de dados disponíveis:

- Modelos e guias de habilidades, competências conhecimentos da Engenharia de Software (SE2004, SWEBOK, SWECOM);

- Projetos pedagógicos e Diretrizes Curriculares Nacionais de Graduação para o curso de Engenharia de Software;

- Validação das listas em consonância com Lacher et al (Lacher, et al., 2015) para a avaliação comportamental de profissionais de software.

Toda esta estrutura de extração e validação foi planejada e executada para obter um objeto final bem estruturado e bibliograficamente embasado. Também, houve uma preocupação que esta lista refletisse as expectativas de instituições e profissionais internacionais, bem como, abrangesse o perfil que as universidades brasileiras almejam desenvolver quanto aos egressos dos seus cursos de Engenharia de Software, em termos não técnicos. Todas essas fontes de informações são importantes dado que o contexto do trabalho são os participantes do programa Ciências sem Fronteiras, ou seja, há a necessidade que os itens levantados estejam alinhados com os currículos de graduação das universidades brasileiras.

O critério base para seleção dos indicadores é que eles representassem competências não técnicas existentes na maior parte das fontes de informação. Aqueles itens que não estavam presentes em pelo menos $50 \%$ dos documentos ficaram de fora da lista obtida pela revisão. Desta forma, os indicadores selecionados demonstram a percepção de um conjunto publicações, inferindo a sua relevância para diversos autores (Simon, 2004).

Com a lista levantada, conforme critérios já mencionados, dois surveys foram planejados e aplicados. Um para aos professores do curso de Engenharia de Software da Universidade de Brasília, para validar e resumir os importantes itens a serem aplicados no questionário dos estudantes. A lista obtida da bibliografia contém muitos itens e não seria viável a sua completa aplicação aos alunos participantes. O outro survey é a principal execução deste trabalho o qual permite tecer análises e conclusões sobre o tema estudado. Toda a estrutura metodológica está resumida na Figura 1. 
V Congresso Brasileiro de Informática na Educação (CBIE 2016)

Anais do XXVII Simpósio Brasileiro de Informática na Educação (SBIE 2016)
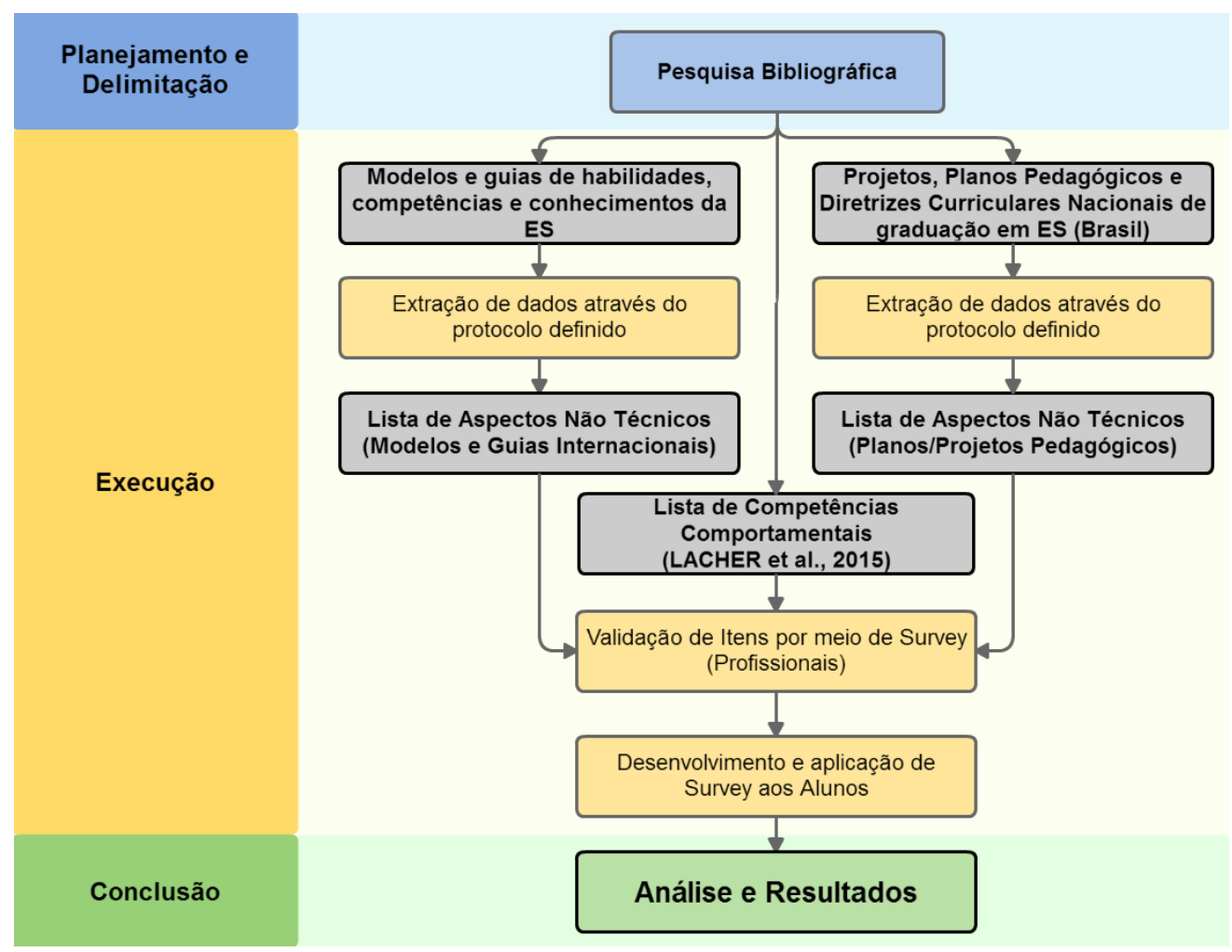

Figura 1. Metodologia do Trabalho

A execução dos protocolos definidos, conforme metodologia definida por Simon (Simon, 2004), resultou em 22 itens relacionados à comunicação, aspectos interpessoais, ética e resolução de problemas.

O questionário foi validado com 12 juízes ou $40 \%$ do corpo docente do curso de Engenharia de Software. Neste questionário foram inseridos 4 itens de controle para verificar o comprometimento e domínio do assunto. Eles foram criados de forma que remetessem a competências não técnicas, porém não sendo itens presentes na bibliografia. Detenção de vocabulário formal (58,3\%), Capacidade de identificar Oportunidades / Empreendedorismo (25\%), Sensibilidade Emocional (50\%), Asseado $(25 \%)$ são os itens de controle, todos obtiverem um percentual de concordância das respostas menor do que o critério definido $(75 \%)$. 12 foram os itens que atenderam o critério definido para a composição do questionário por parte dos estudantes. Desta forma, o questionário final aplicado a população do estudo foi composto destes 12 itens validados, um campo aberto para captação de outras respostas não presente na lista e também 4 questões demográficas.

O questionário final apresentou aos respondentes um cenário no qual descrevia a experiência que o programa buscava proporcionar (graduação em uma instituição de ensino no exterior, participação de projetos e estágios, etc) e pedia que avaliassem em uma escala de 1 a 5 (Likert), como percebiam o desenvolvimento dos 12 indicadores, Q1 a Q12 da Tabela 1, em detrimento da vivência obtida enquanto bolsista do CsF. A implementação e a aplicação do questionário foi feita via Monkey Survey ${ }^{l}$. O questionário foi enviado a 47 estudantes do curso de Engenharia de Software que haviam participado do $\mathrm{CsF}$ e destes 35 estudantes $(\mathrm{N}=35)$ responderam.

\footnotetext{
${ }^{1}$ URL: http://www.surveymonkey.com
} 
V Congresso Brasileiro de Informática na Educação (CBIE 2016)

Anais do XXVII Simpósio Brasileiro de Informática na Educação (SBIE 2016)

Tabela 1: Itens do questionário com indicadores de competências não técnicas.

\begin{tabular}{|l|l|l|}
\hline Q1 & Comunicação Oral & Likert (5) \\
\hline Q2 & Comunicação escrita & Likert (5) \\
\hline Q3 & Sensibilidade cultural & Likert (5) \\
\hline Q4 & Domínio de uma língua estrangeira & Likert (5) \\
\hline Q5 & Sensibilidade social & Likert (5) \\
\hline Q6 & Habilidade de trabalhar em equipe & Likert (5) \\
\hline Q7 & Pensamento crítico & Likert (5) \\
\hline Q8 & Habilidade em obter novos conhecimentos & Likert (5) \\
\hline Q9 & Capacidade em executar pesquisas & Likert (5) \\
\hline Q10 & Organização & Likert (5) \\
\hline Q11 & Responsabilidade & Likert (5) \\
\hline Q12 & Habilidade em gerir o tempo & Likert (5) \\
\hline Q13 & Outro (Campo aberto) & Texto \\
\hline Q14 & Participou Estágio/Pesquisa & Sim/Não \\
\hline Q15 & Acredita que o CsF contribuiu para aspectos técnicos & Sim/Não \\
\hline Q16 & Acredita que o CsF contribuiu para aspectos não técnicos & Sim/Não \\
\hline Q17 & Semestre & Num: 1 a 10 \\
\hline
\end{tabular}

A escala utilizada foi primordialmente a Likert de 5 valores, com as seguintes opções: 1 - não contribuiu, 2- pouco contribuiu, 3 - neutro (indefinido), 4 - contribuiu e 5 - contribuiu bastante. Alguns itens demográficos foram inseridos no questionário: Q14 a Q17.

\section{Análise dos resultados}

$\mathrm{Na}$ fase de análise, em virtude do questionário ser mais amplo, foram considerados apenas os itens aderentes à avaliação dos critérios não técnicos que poderiam impactar na formação de equipe para desenvolvimento de softwares educativos: Sensibilidade social (Q5), sensibilidade cultural (Q3), comunicação oral (Q1), comunicação escrita (Q2), habilidade de trabalhar em equipe (Q6), domínio de uma língua estrangeira (Q4), pensamento crítico (Q7) e habilidade em obter novos conhecimentos (Q8). Também foram considerados os itens Q15 e Q16.

A fim de analisar a confiabilidade dos itens selecionados utilizou-se o fator de Alfa de Cronbach (Cronbach, 1951). O resultado 0,70 indica que o conjunto dos itens selecionados tem validade estatística.

Da análise do coeficiente de correlação de Pearson e considerando os resultados com significância menor que 0,05 , obtiveram-se as seguintes relações:

- A comunicação escrita (Q2) e a sensibilidade cultural (Q3), indicando uma percepção forte dos respondentes que no CsF a comunicação escrita está diretamente ligada à sensibilidade cultural;

- A comunicação Oral (Q1) e o domínio de uma língua estrangeira (Q4), indicando que o domínio da língua é uma condição direta para o desenvolvimento da comunicação oral; e, 
V Congresso Brasileiro de Informática na Educação (CBIE 2016)

Anais do XXVII Simpósio Brasileiro de Informática na Educação (SBIE 2016)

- O domínio de uma língua estrangeira (Q4) e habilidade em obter novos conhecimentos (Q8), indicando que a habilidade de aprender no CsF está diretamente ligado ao domínio da língua.

Das respostas aos itens Q1 a Q8, foi feito um estudo para verificar quais itens foram mais impactados pela participação do estudante no CsF. O resultado está na Tabela 2.

Tabela 2: Resultados médios para os indicadores.

\begin{tabular}{|l|c|c|}
\cline { 2 - 3 } \multicolumn{1}{c|}{} & Média & Desvio padrão \\
\hline Q4 & 4,882 & 0,104 \\
\hline Q3 & 4,647 & 0,228 \\
\hline Q8 & 4,324 & 0,337 \\
\hline Q1 & 4,294 & 0,678 \\
\hline Q7 & 4,294 & 0,443 \\
\hline Q2 & 3,912 & 0,904 \\
\hline Q5 & 3,824 & 1,028 \\
\hline Q6 & 3,265 & 1,136 \\
\hline
\end{tabular}

A Tabela 2 foi dividida em duas partes: Em verde, os itens com votação acima de 4 (quatro) e desvio padrão abaixo de 1 (um) indicando um forte impacto com concordância alta entre os respondentes e, em amarelo, o restante dos itens. Dos itens na parte superior destacam-se o domínio de uma língua estrangeira (Q4) e a sensibilidade cultural (Q3) como valores expressivos e baixo desvio padrão.

Do segundo grupo cabe uma observação com relação à comunicação escrita (Q2) e sensibilidade social (Q5) cujos valores estão próximo do valor 4 (quatro), porém apresentam um desvio padrão alto. Isto indica que os respondentes não estavam muito seguros da contribuição do $\mathrm{CsF}$, apesar de entenderem a relevância dos itens. A distribuição das respostas para estes dois itens está na Tabela 3.

Tabela 3: Distribuição para a comunicação escrita e a sensibilidade social.

Comunicação escrita

\begin{tabular}{|c|c|c|}
\hline Valor & Qt & \% \\
\hline 1 & 0 & $0,00 \%$ \\
\hline 2 & 4 & $11,76 \%$ \\
\hline 3 & 5 & $14,71 \%$ \\
\hline 4 & 15 & $44,12 \%$ \\
\hline 5 & 10 & $29,41 \%$ \\
\hline
\end{tabular}

Sensibilidade social

\begin{tabular}{|c|c|c|}
\hline Valor & Qt & \% \\
\hline 1 & 1 & $2,94 \%$ \\
\hline 2 & 3 & $8,82 \%$ \\
\hline 3 & 6 & $17,65 \%$ \\
\hline 4 & 15 & $44,12 \%$ \\
\hline 5 & 9 & $26,47 \%$ \\
\hline
\end{tabular}

Pela distribuição das respostas, observa-se que houve uma maior concentração de resposta nos valores 4 (quatro) e 5 (cinco), em ambos os casos. Respectivamente, 25 e 24 de 34 respostas, para a comunicação escrita e a sensibilidade social. Isto indica que há uma tendência de resposta onde os estudantes entendem que estes dois indicadores foram muito influenciados pela participação no CsF. 
V Congresso Brasileiro de Informática na Educação (CBIE 2016)

Anais do XXVII Simpósio Brasileiro de Informática na Educação (SBIE 2016)

Por fim, observa-se que a habilidade de trabalhar em equipe (Q6) não foi considerado como uma característica desenvolvida no CsF. Nem pela média, nem pela distribuição que foi em torno do valor 3 (três), indicam um aspecto neutro.

\section{Conclusão}

Este trabalho se propôs a analisar as competências não técnicas adquiridas pelos estudantes que participaram do programa Ciência sem Fronteiras, dado que algumas destas competências são características importantes na formação de profisssionais de desenvolvimento de software que atuarão em equipes de desenvolvimento de aplicativos educacionais.

As competências não técnicas foram obtidas a partir de levantamento bibliográfico das competências não técnicas dos cursos de Engenharia de Software. Foram elencados 22 itens, os quais passaram por uma homologação por uma equipe de juízes (12 professores do curso). Foram filtradas as mais expressivas: 12 indicadores a serem aplicados aos 47 participantes do CsF do curso. Destes 12 indicadores foram selecionados oito: Sensibilidade social, sensibilidade cultural, comunicação oral, comunicação escrita, habilidade de trabalhar em equipe, domínio de uma língua estrangeira, pensamento crítico e habilidade em obter novos conhecimentos. Estes indicadores são algumas das competências esperadas de um profissional que desenvolva aplicativos educacionais.

Verificou-se que sete dos indicadores estão presentes na formação do profissional em Engenharia de Software que participou do CsF. A exceção foi a habilidade de trabalhar em equipe. Isto demonstra uma tendência destes profissionais a serem melhor qualificados para atuar no desenvolvimento de aplicativos educacionais, dado o desenvolvimento de competências que aguçam a sensibilidade necessária para este tipo de dinâmica do aprendizado. Nesta linha de pensamento destaca-se o segundo indicador mais valorado: sensibilidade cultural, importante característica para $\mathrm{o}$ desenvolvimento de software na área educacional.

Como trabalho futuro, a equipe responsável por este trabalho tem acompanhado os egressos verificando o quanto a participação no CsF impactou nas suas carreiras e, em especial, daqueles que foram desenvolver software educacionais.

\section{Referências}

Barreto, R. P., Silva, P. H., Bezerra, M. G., \& Jesus, M. S. (2013). Análise política quanto à eficiência do programa ciências sem Fronteiras: relatos IFRN e UFRN. Anais do IX Congresso de Iniciação Científica do IFRN, (pp. 2240 - 2248). Currais Novos.

Cronbach, L. J. (1951). Coefficient alpha and the internal structure of tests. Psychometrika, 16(3), pp. 297-330.

Governo Brasil. (2011). Decreto $N^{o} 7.642$ - Institui o Programa Ciências sem Fronteiras. Acesso em Maio de 2016, disponível em Casa Civil: http://www.planalto.gov.br/ccivil_03/_Ato2011-2014/2011/Decreto/D7642.htm

Governo Brasil. (2014). Painel de Controle do Programa Ciência sem Fronteiras. Acesso em Maio de 2016, disponível em Ciências sem Fronteiras: http://www.cienciasemfronteiras.gov.br/web/csf/painel-de-controle 
V Congresso Brasileiro de Informática na Educação (CBIE 2016)

Anais do XXVII Simpósio Brasileiro de Informática na Educação (SBIE 2016)

IEEE Computer Society and ACM. (2004). Software engineering 2004 - Curriculum Guidelines for Undergraduate Degree Programs in Software Engineering (Vol. Computing Curricula Series). IEEE and ACM.

Lacher, L. L., Walia, G. S., Fagerholm, F., Pagels, M., Nygard, K., \& J. M. (2015). A behavior marker tool for measurement of the non- technical skills of software professionals: An empirical investigation. The 27th International Conference on Software Engineering and Knowledge Engineering, (pp. 478-483). Pittsburgh, USA.

Ministério da Educação. (2002). Resolução CNE/CES nº 11 - Diretrizes Curriculares Nacionais para os cursos de graduação em Engenharia. Brasília, Brasil.

Ministério da Educação. (2012). Parecer CNE/CES no 136 - Diretrizes Curriculares Nacionais para os cursos de graduação em Computação. Brasília, DF, Brasil.

Neto, J. C., Reinehr, S., \& Malucelli, A. (2015). Processo de Desenvolvimento de Software: uma Análise Exploratória com Profissionais que Desenvolvem Jogos Eletrônicos Educacionais. Anais do XXVI Simpósio Brasileiro de Informática na Educação (SBIE 2015), (pp. 289-298).

Pasquali, L. (2011). Psicometria: Teoria dos testes na Psicologia e na Educação (4. ed. ed.). Rio de Janeiro: Vozes.

Rivera-Ibarra, J. G., Rodríguez-Jacobo, J., \& Serrano-Vargas, M. A. (2010). Competency Framework for Software Engineers. 23rd IEEE Conference on Software Engineering Education and Training, (pp. 33-40). Pittsburgh - EUA.

Santos, R. E., Magalhães, C. V., Correia-Neto, J. S., Souza, E. P., \& Vilar, G. (2014). Ferramentas, métodos e experiências no ensino de Engenharia de Software: um mapeamento sistemático. Anais do XXV Simpósio Brasileiro de Informática na Educação (SBIE 2014), (pp. 544-548).

Simon, F. (2004). Habilidades e competências em engenharia: Criação e validação de um instrumento. Campinas - SP: Unicamp.

SWEBOK. (2014). Guide to the Software Engineering Body of Knowledge, Version 3.0. (P. Bourque, \& R. Fairley, Eds.) IEEE Computer Society.

SWECOM. (2015). Software engineering competency model. IEEE Computer Society.

Wirth, N. (2008). Brief History of Software Engineering. In: IEEE Annals of the History of Computing (pp. 32-39). IEEE.

Wohlin, C., Runeson, P., Höst, M., Ohlsson, M. C., Regnell, B., \& Wesslén, A. (2012). Experimentation in Software Engineering. Springer-Verlag. 